\title{
Supersymmetric Hamilton Operator and Entanglement
}

\author{
Willi-Hans Steeb and Yorick Hardy \\ International School for Scientific Computing, University of Johannesburg, Auckland Park 2006, \\ South Africa
}

Reprint requests to Prof. W.-H. S; E-mail: WHS@NA.RAU.AC.ZA

Z. Naturforsch. 61a, 139 - 140 (2006); received February 22, 2006

We study the entanglement of Fermi particles of a supersymmetric Hamilton operator given by a simple Fermi-Bose system.

Key words: Supersymmetric Hamiltonian; Entanglement; Fermi Operators; Bose Operators.

Entanglement has been studied in detail for finitedimensional quantum systems and to a lesser extent for infinite-dimensional quantum systems (see $[1,2]$ and references therein). Here we study the entanglement for states of a supersymmetric Hamilton operator [3] given by Bose operators $b^{\dagger}, b$ and Fermi operators with spin up and spin down, i.e. $c_{\uparrow}^{\dagger}, c_{\downarrow}^{\dagger}, c_{\uparrow}, c_{\downarrow}$. Let

$$
Q:=b \otimes c_{\uparrow}^{\dagger} c_{\downarrow}^{\dagger}
$$

be a linear operator, where $b$ is a Bose annihilation operator, $c_{\uparrow}^{\dagger}$ is a Fermi creation operator with spin up, $c_{\downarrow}^{\dagger}$ is a Fermi operator with spin down and $\otimes$ the tensor product [4]. Since $c_{\sigma}^{\dagger} c_{\sigma}^{\dagger}=0, \sigma \in\{\uparrow, \downarrow\}$ we find that $Q^{2}=0$. We define the supersymmetric Hamilton operator $\hat{H}$ as

$$
\hat{H}:=\left[Q, Q^{\dagger}\right]_{+} \equiv Q Q^{\dagger}+Q^{\dagger} Q .
$$

From (1) we obtain $Q^{\dagger}=b^{\dagger} \otimes c_{\downarrow} c_{\uparrow}$. Let $\hat{n}_{B}:=b^{\dagger} b$, $\hat{n}_{\uparrow}:=c_{\uparrow}^{\dagger} c_{\uparrow}, \hat{n}_{\downarrow}:=c_{\downarrow}^{\dagger} c_{\downarrow}$ be the number operators. Applying $\left[b, b^{\dagger}\right]=I_{\mathrm{B}}$ and $\left[c_{\sigma}, c_{\sigma^{\prime}}^{\dagger}\right]_{+}=I_{\mathrm{F}} \delta_{\sigma, \sigma^{\prime}}$ we arrive at

$$
\hat{H}=\left(2 \hat{n}_{B}+I_{B}\right) \otimes \hat{n}_{\uparrow} \hat{n}_{\downarrow}+\hat{n}_{B} \otimes\left(I_{F}-\hat{n}_{\uparrow}-\hat{n}_{\downarrow}\right),
$$

where $I_{B}$ is the identity operator in the Hilbert space $\mathcal{H}_{B}$ of the Bose operators and $I_{F}$ is the identity operator in the Hilbert space $\mathcal{H}_{F}$ of the Fermi operators. Straightforward calculation yields $[\hat{H}, Q]=0$ and $\left[\hat{H}, Q^{\dagger} Q\right]=0$. Thus the three operators $\hat{H}, Q, Q^{\dagger} Q$ may be diagonalized simultaneously. Let $|n\rangle$ be the number states (Fock states), where $n=0,1,2, \ldots$ and $\langle n \mid n\rangle=1$.
For the Fermi operators we use the matrix representation [4]

$$
c_{\uparrow}^{\dagger}=\frac{1}{2} \sigma_{+} \otimes I_{2}, \quad c_{\downarrow}^{\dagger}=\frac{1}{2} \sigma_{z} \otimes \sigma_{+} .
$$

Thus

$$
c_{\uparrow}=\frac{1}{2} \sigma_{-} \otimes I_{2}, \quad c_{\downarrow}=\frac{1}{2} \sigma_{z} \otimes \sigma_{-}
$$

and

$$
c_{\uparrow}^{\dagger} c_{\downarrow}^{\dagger}=-\frac{1}{4} \sigma_{+} \otimes \sigma_{+} .
$$

Thus the Fermi operators act in the Hilbert space $\mathbf{C}^{4}$. It follows that

$$
\begin{gathered}
\hat{n}_{\uparrow}=c_{\uparrow}^{\dagger} c_{\uparrow}=\left(\begin{array}{ll}
1 & 0 \\
0 & 0
\end{array}\right) \otimes I_{2}, \quad \hat{n}_{\downarrow}=c_{\downarrow}^{\dagger} c_{\downarrow}=I_{2} \otimes\left(\begin{array}{ll}
1 & 0 \\
0 & 0
\end{array}\right), \\
\hat{n}_{\uparrow} \hat{n}_{\downarrow}=\left(\begin{array}{ll}
1 & 0 \\
0 & 0
\end{array}\right) \otimes\left(\begin{array}{ll}
1 & 0 \\
0 & 0
\end{array}\right) .
\end{gathered}
$$

Then a basis in the product Hilbert space is given by

$$
\begin{aligned}
& |n\rangle \otimes\left(\begin{array}{l}
1 \\
0
\end{array}\right) \otimes\left(\begin{array}{l}
1 \\
0
\end{array}\right), \quad|n\rangle \otimes\left(\begin{array}{l}
1 \\
0
\end{array}\right) \otimes\left(\begin{array}{l}
0 \\
1
\end{array}\right), \\
& |n\rangle \otimes\left(\begin{array}{l}
0 \\
1
\end{array}\right) \otimes\left(\begin{array}{l}
1 \\
0
\end{array}\right), \quad|n\rangle \otimes\left(\begin{array}{l}
0 \\
1
\end{array}\right) \otimes\left(\begin{array}{l}
0 \\
1
\end{array}\right),
\end{aligned}
$$

where $n=0,1,2, \ldots$ Now we obtain

$$
\hat{H}|n\rangle \otimes\left(\begin{array}{l}
1 \\
0
\end{array}\right) \otimes\left(\begin{array}{l}
1 \\
0
\end{array}\right)=(n+1)|n\rangle \otimes\left(\begin{array}{l}
1 \\
0
\end{array}\right) \otimes\left(\begin{array}{l}
1 \\
0
\end{array}\right) .
$$

This is an eigenvalue equation with eigenvalue $n+1$, where $n=0,1,2, \ldots$. Furthermore

$$
\hat{H}|n\rangle \otimes\left(\begin{array}{l}
1 \\
0
\end{array}\right) \otimes\left(\begin{array}{l}
0 \\
1
\end{array}\right)=0,
$$




$$
\hat{H}|n\rangle \otimes\left(\begin{array}{l}
0 \\
1
\end{array}\right) \otimes\left(\begin{array}{l}
1 \\
0
\end{array}\right)=0 .
$$

Both states have eigenvalue 0 . Finally

$$
\hat{H}|n\rangle \otimes\left(\begin{array}{l}
0 \\
1
\end{array}\right) \otimes\left(\begin{array}{l}
0 \\
1
\end{array}\right)=n|n\rangle \otimes\left(\begin{array}{l}
0 \\
1
\end{array}\right) \otimes\left(\begin{array}{l}
0 \\
1
\end{array}\right)
$$

with eigenvalue $n$, where $n=0,1,2, \ldots$ Thus the lowest eigenvalue is 0 . The Bell states are given by

$$
\begin{aligned}
& \left|\Phi^{+}\right\rangle=\frac{1}{\sqrt{2}}\left(\left(\begin{array}{l}
1 \\
0
\end{array}\right) \otimes\left(\begin{array}{l}
1 \\
0
\end{array}\right)+\left(\begin{array}{l}
0 \\
1
\end{array}\right) \otimes\left(\begin{array}{l}
0 \\
1
\end{array}\right)\right), \\
& \left|\Phi^{-}\right\rangle=\frac{1}{\sqrt{2}}\left(\left(\begin{array}{l}
1 \\
0
\end{array}\right) \otimes\left(\begin{array}{l}
1 \\
0
\end{array}\right)-\left(\begin{array}{l}
0 \\
1
\end{array}\right) \otimes\left(\begin{array}{l}
0 \\
1
\end{array}\right)\right), \\
& \left|\Psi^{+}\right\rangle=\frac{1}{\sqrt{2}}\left(\left(\begin{array}{l}
1 \\
0
\end{array}\right) \otimes\left(\begin{array}{l}
0 \\
1
\end{array}\right)+\left(\begin{array}{l}
0 \\
1
\end{array}\right) \otimes\left(\begin{array}{l}
1 \\
0
\end{array}\right)\right), \\
& \left|\Psi^{-}\right\rangle=\frac{1}{\sqrt{2}}\left(\left(\begin{array}{l}
1 \\
0
\end{array}\right) \otimes\left(\begin{array}{l}
0 \\
1
\end{array}\right)-\left(\begin{array}{l}
0 \\
1
\end{array}\right) \otimes\left(\begin{array}{l}
1 \\
0
\end{array}\right)\right) .
\end{aligned}
$$

Consider the product states of the number states and the Bell states. Applying the Hamilton operator we find

$$
\begin{aligned}
\hat{H}|n\rangle \otimes\left|\Phi^{+}\right\rangle & =n|n\rangle \otimes\left|\Phi^{+}\right\rangle+\frac{1}{\sqrt{2}}|n\rangle \otimes\left(\begin{array}{l}
1 \\
0
\end{array}\right) \otimes\left(\begin{array}{l}
1 \\
0
\end{array}\right), \\
\hat{H}|n\rangle \otimes\left|\Phi^{-}\right\rangle & =n|n\rangle \otimes\left|\Phi^{-}\right\rangle+\frac{1}{\sqrt{2}}|n\rangle \otimes\left(\begin{array}{l}
1 \\
0
\end{array}\right) \otimes\left(\begin{array}{l}
1 \\
0
\end{array}\right), \\
\hat{H}|n\rangle \otimes\left|\Psi^{+}\right\rangle & =0, \\
\hat{H}|n\rangle \otimes\left|\Psi^{-}\right\rangle & =0 .
\end{aligned}
$$

[1] W.-H. Steeb and Y. Hardy, Problems and Solutions in Quantum Computing and Quantum Information, World Scientific, Singapore 2006.

[2] Y. Hardy and W.-H. Steeb, Int. J. Theor. Phys. 43, 2207 (2004).
Consider now the unitary operator $U(t)=\exp (-\mathrm{i} \hat{H} t)$. Then we obtain

$$
\begin{aligned}
& U(t)|n\rangle \otimes\left(\begin{array}{l}
1 \\
0
\end{array}\right) \otimes\left(\begin{array}{l}
1 \\
0
\end{array}\right)=\mathrm{e}^{-\mathrm{i} t(n+1)}|n\rangle \otimes\left(\begin{array}{l}
1 \\
0
\end{array}\right) \otimes\left(\begin{array}{l}
1 \\
0
\end{array}\right), \\
& U(t)|n\rangle \otimes\left(\begin{array}{l}
0 \\
1
\end{array}\right) \otimes\left(\begin{array}{l}
0 \\
1
\end{array}\right)=\mathrm{e}^{-\mathrm{i} t n}|n\rangle \otimes\left(\begin{array}{l}
0 \\
1
\end{array}\right) \otimes\left(\begin{array}{l}
0 \\
1
\end{array}\right)
\end{aligned}
$$

and

$$
\begin{aligned}
& U(t)|n\rangle \otimes\left(\begin{array}{l}
1 \\
0
\end{array}\right) \otimes\left(\begin{array}{l}
0 \\
1
\end{array}\right)=|n\rangle \otimes\left(\begin{array}{l}
1 \\
0
\end{array}\right) \otimes\left(\begin{array}{l}
0 \\
1
\end{array}\right), \\
& U(t)|n\rangle \otimes\left(\begin{array}{l}
0 \\
1
\end{array}\right) \otimes\left(\begin{array}{l}
1 \\
0
\end{array}\right)=|n\rangle \otimes\left(\begin{array}{l}
0 \\
1
\end{array}\right) \otimes\left(\begin{array}{l}
1 \\
0
\end{array}\right) .
\end{aligned}
$$

It follows that

$$
\begin{aligned}
U(t)|n\rangle \otimes\left|\Phi^{+}\right\rangle= & \frac{1}{\sqrt{2}} \mathrm{e}^{-\mathrm{i} t n}|n\rangle \\
& \otimes\left(\mathrm{e}^{-\mathrm{i} t}\left(\begin{array}{l}
1 \\
0
\end{array}\right) \otimes\left(\begin{array}{l}
1 \\
0
\end{array}\right)+\left(\begin{array}{l}
0 \\
1
\end{array}\right) \otimes\left(\begin{array}{l}
0 \\
1
\end{array}\right)\right), \\
U(t)|n\rangle \otimes\left|\Phi^{-}\right\rangle= & \frac{1}{\sqrt{2}} \mathrm{e}^{-\mathrm{i} t n}|n\rangle \\
& \otimes\left(\mathrm{e}^{-\mathrm{i} t}\left(\begin{array}{l}
1 \\
0
\end{array}\right) \otimes\left(\begin{array}{l}
1 \\
0
\end{array}\right)-\left(\begin{array}{l}
0 \\
1
\end{array}\right) \otimes\left(\begin{array}{l}
0 \\
1
\end{array}\right)\right),
\end{aligned}
$$

and $U(t)|n\rangle \otimes\left|\Psi^{+}\right\rangle=|n\rangle \otimes\left|\Psi^{+}\right\rangle, U(t)|n\rangle \otimes\left|\Psi^{-}\right\rangle=$ $|n\rangle \otimes\left|\Psi^{-}\right\rangle$. Thus the states $|n\rangle \otimes\left|\Psi^{+}\right\rangle$and $|n\rangle \otimes\left|\Psi^{-}\right\rangle$ do not change under the unitary transformation. The Fermi part of the state $U(t)|n\rangle \otimes\left|\Phi^{ \pm}\right\rangle$is also a Bell state. There is a continuous oscillation between $|n\rangle \otimes$ $\left|\Phi^{+}\right\rangle$and $|n\rangle \otimes\left|\Phi^{-}\right\rangle$with periodicity $2 \pi$.

[3] W.-H. Steeb, Problems and Solutions in Theoretical and Mathematical Physics, Vol. II: Advanced Level, 2nd ed., World Scientific, Singapore 2003.

[4] W.-H. Steeb, Matrix Calculus and Kronecker Product with Applications and C++ Programs, World Scientific, Singapore 1997. 